\section{PHOTOGRAPHIC ANALYSIS OF MOTION*}

\section{By E. R. DAVIES}

\section{Kodak Research Laboratories}

$\mathrm{T}$ -DAY we turn naturally to photography to analyse movements which are too rapid for our eyes to follow. If we tend to take this powerful weapon too much for granted, it must be remembered that there were formidable experimental difficulties facing the investigator before the advent of photography. Marey, while director of the Physiological Station in Paris, devised a most ingenious way of studying the movements of a horse. Pressure chambers fitted to the underside of the horse's hooves were connected by pneumatic tubes to a recording mechanism held by the rider, which registered the time of duration of contact of each hoof with the ground. Marey did much painstaking work along these lines between 1860 and 1880 .

Muybridge, a native of Kingston-on-Thames, was conducting a photographic survey of the Californian coast in 1872 when he was appealed to by Governor Sandford to settle a controversy which was raging in San Francisco and which was aroused by Marey's analysis of the movements of a horse. The question was whether a trotting horse ever had all its feet free of the ground simultaneously. Muybridge set himself the task of obtaining photographic proof and achieved immediate success. He used a camera fitted with what may be loosely described as an instantaneous shutter and, taking photographs at random, soon obtained one which showed all the horse's feet free from the ground. The controversy was settled; but, more important, the direction of Muybridge's life's work was settled also.

For the exhaustive investigation of animal locomotion, upon which he soon embarked, Muybridge erected an elaborate apparatus on the racecourse at Palo Alto, near San Francisco. A battery of twentyfour cameras was arranged along one side of the track. Across the track was stretched a number of threads each connected to the shutter on one of the twenty. four cameras. The camera shutter was in the form of a guillotine urged downwards by a spring, and was released by an electro-magnetic relay on the breaking of the thread. In this way, as the horse passed in front of the cameras, it broke the threads in succession, and a series of instantaneous photographs was obtained. The time of exposure given by the shutters was of the order of $1 / 1,000$ sec., and it is remarkable that Muybridge was able to obtain sufficient exposure with the photographic materials available to him. This was the time of the wet-plate process, and, compared with modern practice, photography was laborious in the extreme. To obtain sufficient exposure, Muybridge arranged a sloping white background to catch the light of the sun. Against this the horse stood out in sharp relief. Even so, his early results were mere silhouettes lacking all detail. With the increasing use of the gelatin dry plate (invented by Maddox in 1871), the photographic process was greatly simplified, and the sensitivity to light was increased many times. In his later work, Muybridge was thus enabled to obtain photographs showing detail. An impression of the scale of his operations will

- Substance of a Friday discourse delivered at the Royal Institution on December 11, 1942. be gained from the statement that he exposed no fewer than 100,000 plates in the two years, 1884 and 1885 .

Muybridge showed how the pictures taken successively by his battery of cameras could be combined to reproduce the appearance of the original movements. He printed his negatives in order on a band of paper. One of his original records was presented to the Royal Institution following the discourse he gave in 1882. The record, which is still preserved at the Institution, is placed in an apparatus known as a zoetrope, which is simply an empty cylinder with vertical slots arranged at equal intervals around the circumference, and it is carried on a vertical axis. On rotating the cylinder the pictures are viewed in succession through the slots and appear to be in motion. Muybridge thus raised a toy of the Victorian drawing-room to the status of a scientific instrument, and used it to demonstrate the correctness of his method of photographic analysis. The zoetrope, and other improved designs for which he was responsible, may be rightly regarded as forerunners of the modern cinematograph projector.

The cinematograph projector and camera were brought a stage closer by Marey, who, in his chronograph, employed a band of film on which successive photographs were taken, and who devised another apparatus for viewing the results by projection on a screen. Marey was a most remarkable man. I have already mentioned his work with pressure chambers and pneumatic tubes. Introduced to the photographic method by Muybridge, he was quick to seize upon its possibilities. His book "Le Mouvement" (published in 1894) reveals him as a brilliant experimentalist. His investigations covered the analysis of the locomotion of many animals, of birds in flight, of insects and even of micro-organisms by means of photography under the microscope. Few developments in high-speed photography were not visualized, and in some part realized, by him.

As typical of his work, I will single out for mention one technique which has proved of lasting value. Marey built a camera in which the shutter was a disk with radial slots. This revolved in front of the camera lens and caused a series of photographs to be taken in rapid succession on the same plate. In place of Muybridge's brilliantly lit background against which the moving object was revealed as a silhouette, Marey used a black background and whitened his object. In this way only the object (and not the background) was recorded during the successive exposures of the plate.

Marey's' rotating disk shutter with its radial slots gave individual exposures of about $1 / 25,000$ sec. This was an advance on Muybridge's shutter time of $1 / 1,000$ sec., but is nevertheless far too long a time for many purposes. Mechanical shutters of any type have obvious limitations due to the inertia of the moving parts. But the problem can be approached from a different angle. It will be obvious that the desired result will be obtained if the camera shutter is left open and the subject illuminated instantaneously. Light flashes can be obtained of extremely short duration, and their use in 'stopping' rapid motion represents a milestone in the road of progress.

Lord Rayleigh used magnesium powder for a slow flash, and the electric spark for a fast flash with a time of duration of the order of one millionth of a second. With this he was successful in photographing a soap film in the act of disrupting - a phenomenon that takes place with extreme rapidity. 


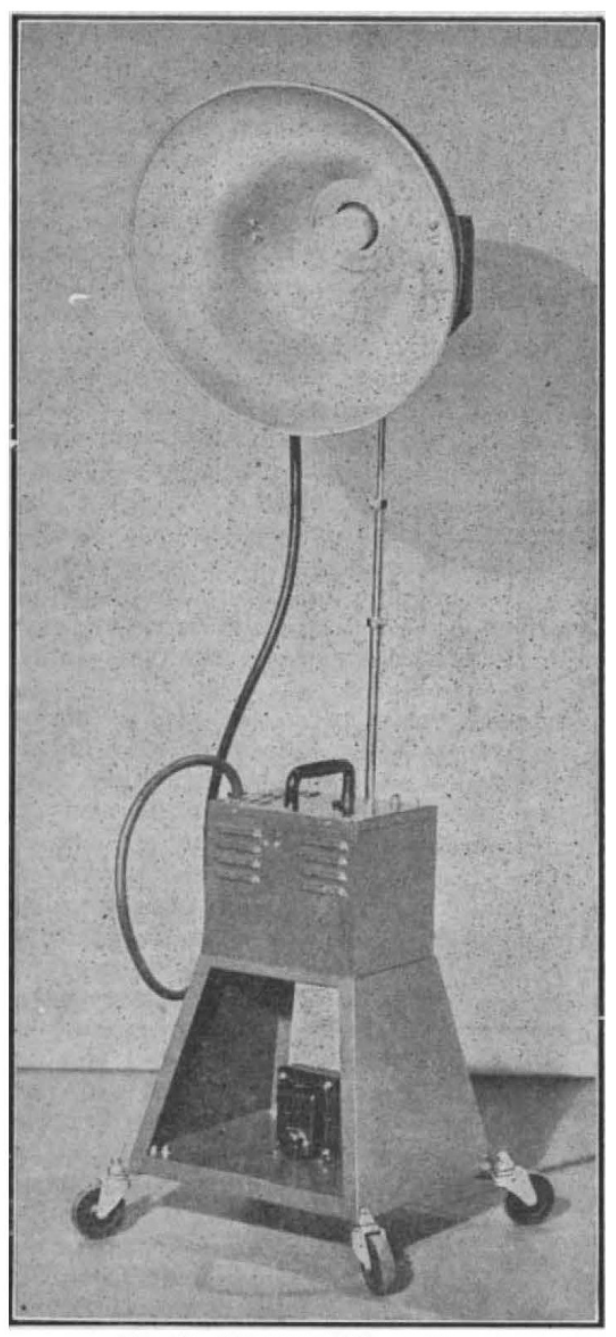

Fig. 1. KodATroN SPEED LAMP.

Prior to this time Worthington had used the electric spark in his beautiful experiments on the formation of drops and the mechanism of splashes*. His early work was not photographic. He observed different phases of the splash made apparently stationary by the light of the flash, and sketched what he saw. For his later investigations on surface tension phenomena he used photography.

The electric spark is fast enough to permit the photography of projectiles in flight. Sir Charles Boys took such photographs around 1890. They were shadow photographs; no camera or lens was used. The bullet passed between the photographic plate and the spark, and thus cast the shadow. In front of the plate, and in the line of flight, were two wires forming a gap in series with the spark gap. As the bullet bridged the gap, the fall in the resistance of the circuit caused the spark to pass and the photograph was obtained. Many associated phenomena are clearly revealed in such photographs. The nose of the bullet forms a bow wave. This travels with the speed of sound in air, about 1,100 ft./sec., and Boys showed how the speed of the bullet can be calculated from the angle between the bow wave and

* Worthington, A. M., "The Splash of a Drop and Allied Phenomena" (Proc. Roy. Inst., May 18, 1894) : "A Study of Splashes" (London, 1908). the line of flight. The bullet, though travelling at more than twice the speed of sound, is revealed with extreme sharpness by the spark, which lasts for less than one millionth of a second. Boys succeeded in measuring the duration of his spark by reflecting it on to a photographic plate by a rapidly revolving mirror and measuring the length of the trace-a method which we still use to-day.

Boys' projectile photographs bear comparison with the most modern work. The chief difference seen in the beautiful photographs published by Quayle of the Bureau of Standards in 1925 is the absence of the synchronizing wires. Quayle fired his spark by means of a microphone actuated by the noise of the explosion. This method was suggested by Dr. Mach of Vienna, whose father, Prof. Mach of Prague, was the first to obtain photographs of bullets in 1881 . Mach, however, was not able to realize this method of synchronization with the apparatus available to him.

So much for the early history of the subject. By the turn of the century the principles of photographic analysis of motion were well understood : the possibilities of the method were fully realized. Advance since that time is largely a story of improvements in technique : improvements in photographic materials, in light sources and, above all, in the development of the cinematograph.

As to photographic materials, it is a story of steady improvement in quality and advance in sensitivity. I thought we had nearly reached finality a few years ago; but the War has brought forth a tremendous effort, and the fastest materials to-day are several times as sensitive as they were only two or three years ago. At a guess-it is little more in the absence of precise data-I should say thàt since Muybridge's time we have seen an increase of at least a hundred times in the speed of photographic materials. That is to say, we can achieve similar results to-day with $1 / 100$ th of the light.

Lenses, too, have improved out of all knowledge. But here again it is difficult to give a precise figure for the effective increase in speed, for the very fast Petzval portrait lens was introduced in 1840 - within a year of the invention of photography. However, it was poorly corrected, and a common aperture in those days was $f / 8$. To-day $f / 1 \cdot 5$ is equally common-an increase in lightgathering power of thirty times.

To turn to light sources: the spark is an admirable way of obtaining extremely short exposures, and is probably unsurpassed when exposures of less than a millionth of a second are required. Apart from projectile photography, however, such extremely short exposures are rarely needed. A large output of light is more often desirable, and the possibilities of the spark in this direction are severely limited.

Recent developments of the gas discharge tube have resulted in a much more generally useful source of light. For the cold

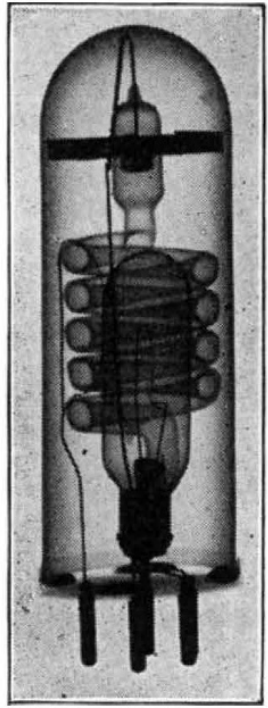

Fig. 2. RADIOGRAPH OF THE GASEOUS DISCHARGE TUBE AND MODELLING IIGHT ASSEMBLY FROM THE KODATRON CHARGE TUBE IS WOUND ROUND THE TUNGSTEN FILAMENT LAMP WHICH IS USED FOR ARRANGING THE LIGHTING ON THE SUBJECT. NOTE (AT THE LEFT) THE TRIGGERING ELECTRODE PASSED ROUND THE COILED DISCHARGE TUBE. SPEED LAMP. THE DIS- 
cathode discharge tube, which represents a most important advance, we are largely indebted to Edgerton, of the Massachusetts Institute of Technology. A commercial version of the cold cathode discharge tube is the Kodatron Speed Lamp (Figs. 1 and 2). This consists of a discharge tube wound in the form of a helix to give a compact source of light, with a frosted glass container and a reflector. The tube is filled with a mixture of rare gases at low pressure and provided with anode and catbode. The Kodatron is self-contained, with a control box in the base of the stand supporting the reflector. On connecting with the 240 volt A.C. supply and switching on, a bank of condensers is charged to 2,000 volts. Nothing happens, since the gas pressure is adjusted to withstand this potential. The tube contains, however, a third electrode and, on depressing a second switch, this conveys a highfrequency pulse to the tube. The gas is ionized; the sparking potential drops to below 2,000 volts; the current passes between the electrodes and is accompanied by a vivid flash of light. The duration of the flash depends upon the condenser capacity. Using the full capacity of $112 \mathrm{mfd}$., the flash lasts for $1 / 5,000$ sec. Within the angle of the reflector the luminous output is of the order of 50 million candlepower; and with a fast film and high-aperture lens, photographs can be taken of objects at a distance of 100 yd. from the lamp.

As this lamp demonstrates, electronics have played their part in the advance in the design of light sources. The application of electronics has also enormously simplified the problems of synchronization. If a beam of light is caused to fall upon a photo-electric cell, the cutting of the beam can be made to fire the flash. A microphone connected with the Kodatron illustrates the principle of synchronization by sound. By varying the distance between the microphone and the source of sound, a time lag can be introduced to the extent of $1 / 1,000 \mathrm{sec}$. per foot. This is often convenient in projectile photography. The spark can be fired at any predetermined interval of time after the explosion and the bullet thus photographed at any required distance from the muzzle.

Dr. Slack, of the Westinghouse Laboratories, has recently devised an X-ray tube which does not contain a heated filament to provide the source of electrons. It functions in much the same way as Edgerton's cold cathode discharge tube. A burst of current through the tube causes an emission of X-rays limited in duration to a millionth of a second. Bullets can be photographed with this apparatus as they pass along the gun barrel.

So far we have been concerned for the most part with the taking of a single photograph : with snatching a fleeting instant from the passage of time to reveal one phase of an event, and with methods whereby we can ensure that we capture the instant of interest. If we wish to study the whole course of an event, we must turn to the cinematograph. In the high-speed cine-camera the pictures are taken at a much greater rate than that used for projection, and so the event is stretched out in time.

In projection at least sixteen pictures must fall on the screen in each second if flicker is to be avoided and the appearance of continuous motion is to be obtained. The modern high-speed cine-camera will take 3,000 pictures per sec. We are thus able to slow down the motion in the ratio of 3,000 to 16 , a factor of about two hundred times. For this reason the high-speed cine-camera is often referred to as a 'time microscope', which, in the, case I have quoted, can be said to have a 'magnification' of two hundred times.

In the ordinary cine-camera the film is moved intermittently. This is impossible at high speeds; the film must run continuously and the desired result must be produced in other ways. Edgerton overcomes the difficulty by using his discharge lamp to illuminate the subject by a succession of flashes to give the separate pictures on the continuously running film. The value of the method to modern industry will be obvious.

In industrial applications, however, it is rare that one can bring the problem to the laboratory. We need readily portable equipment, such as the Kodak High Speed camera. The subject is illuminated in the ordinary way, that is, continuously. The film also runs continuously through the focal plane of the lens. Between the lens and the film a block of glass rotates to form a shutter and to provide optical compensation for the film movement (Fig. 3). The glass block is fitted with vanes which form part of the surface of a cylinder and act as shutters. During each revolution, light from the lens is admitted to the film and a photograph is taken. The light is refracted downwards by the glass block as it passes through the vertical position in such a way that the image moves with the film and relative motion is avoided.

At a frequency of 3,000 pictures per second-the maximum speed of the camera-the time of exposure of each photograph is $1 / 10,000$ sec. At this frequency the film is travelling at $75 \mathrm{ft} . / \mathrm{sec}$., and, were it not for the optical compensation, the exposure time would have to be reduced to a few millionths of a second to reduce the effects of film movement to negligible proportions. These conditions can be met by Edger. ton's flashing lamp technique, but only with sacrifice of illumination. For the analysis of many phenomena, the most important requirement is a high frequency of picture taking: given this, the exposures of the separate pictures can be relatively long, and the ability to expose for so long as 1/10,000 sec., conferred by optical compensation of film movement, greatly simplifies illumination problems which, however, remain formidable enough. Illumination of the subject to a level approximating to sunlight on a fine summer day is necessary.

The camera is comparatively light and compact. It takes a 100-ft. roll of film of the type normally used for amateur cinematography. The base of the camera has been fitted with a timing device. This consists of an electrically controlled 1,000-cycle tuming fork, a small lamp and an optical system which throws an image of the lamp filament on to the edge of the film. The light beam is interrupted by shutter blades attached to the prongs of the fork to give a series of dots along the edge of the film at $1 / 1,000 \mathrm{sec}$. intervals.

In the ideal case the photography is simple. However, each problem usually presents special difficulties which must be overcome. A typical example is afforded by an investigation which was carried out of the performance of a drop forging hammer. The hammer weighed 8 tons and the whole machine was based on some 500 tons of concrete. The users were concerned by the slight displacements which took place in this massive base. It was desired to know the extent of movement of the anvil under impact. Observers had put the figure at between 1 in. and 2 in., and we were asked to carry out measurements 


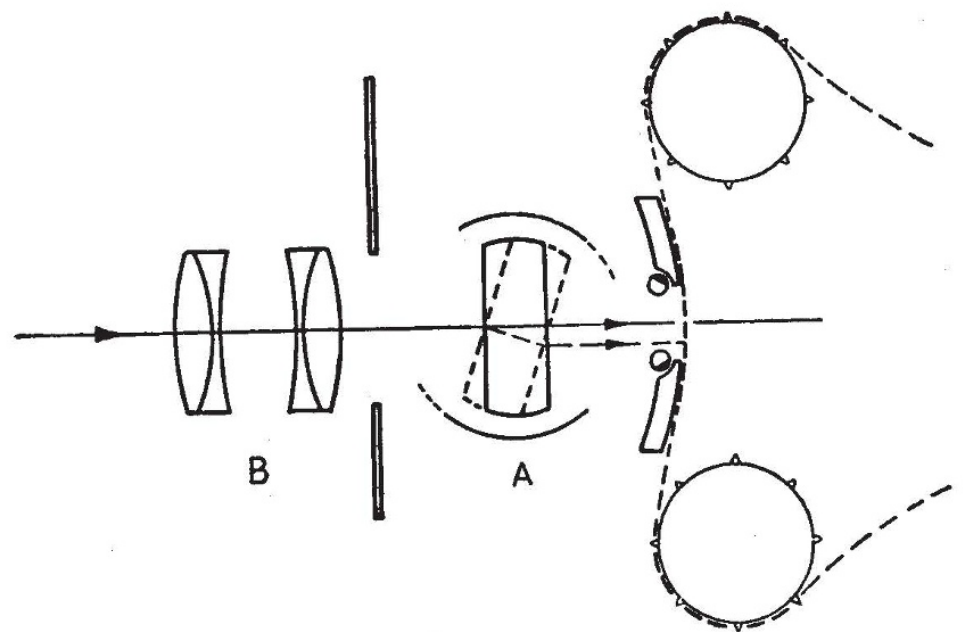

Fig. 3. SCHEMATIC DIAGRAM OF A NON-INTERMTTTENT CINE-CAMGRA WITH OPTICAL COMPENSATION.

by high-speed photography. The problem would have been simple but for two facts. First, on impact there was a cloud of smoke and flame from the fluxes used in the mould, and the anvil was largely obliterated. Secondly, everything in the vicinity rocked as if by an earthquake, and there was no possibility of insulating the camera from the violent vibrations. Thus it was necessary to include in each photograph some fixed object to which the displacement of the anvil could be referred. This was found in a lowfrequency pendulum suspended at the centre of a stretched elastic cord. Vanes attached to the bob of the pendulum dipped in an oil bath and prevented any other than vertical motion. The low frequency of the pendulum - of the order of 2 sec.-prevented it from moving until long after the impact. Thus movements of the anvil only were recorded. A vane on top of the pendulum partly obliterated the image of a bright light source reflected into the camera from a prism which was fixed in the anvil. When the anvil moved, the slit image of the light source widened and narrowed, and these movements were photographed. An analysis of the photographs showed that the movement ended in 1/200 sec. and the maximum displacement was $1 / 20$ in.-five times less than the lowest visual estimate.

Sometimes it is the analysis of the records, and not the photography, which presents unusual difficulties. An ingenious method to overcome them is due to the L.M.S. Railway Research Laboratory. In an investigation of the influence of the profile of the carriage wheel on the smoothness of running of a train, it was desired to measure the sideways motion of the wheel on the rail during travel. Arrangements were made to take a cine-picture of the point of contact of rail and wheel. On each of the many thousands of photographs obtained, the displacement had to be measured-an almost impossible task by ordinary means. The special apparatus which was devised allowed the measurements to be made easily and quickly. In place of the usual cine-projection screen, a 'recording screen' was used; and during projection of the film the recording paper was moved synchronously with the passage of the film through the projector. One observer by means of a lever was able to move the whole screen bodily sideways to reep the image of the rail against a datum line. Another observer kept an index on the image of the wheel and made a trace on the paper. The distance of this from the datum line gave the required information--a kind of 'indicator diagram' on the recording screen which could be evaluated mathematically.

These two examples of the way in which physical devices are used to overcome experimental difficulties must suffice. I am very conscious of many omissions. I have not mentioned Sir Charles Boys' ingenious lightning eamera which, in the hands of Schonland and others, has been so fruitful in the analysis of the lightning discharge. I have not mentioned the large amount of work on flame propagation which has resulted in the unravelling of many of the mysteries of combustion by Ellis, Bone and Frazer, to mention three outstanding workers in Great Britain. My excuse must be that the possibilities of wedding experimental ingenuity to photography are almost boundless. Another omission is the analysis of very slow phenomena. Perhaps here I may plead that there is no problem. There is no difficulty in taking photographs with intervals of minutes or days. The film is projected at normal speed and the passage of time is telescoped. In this way much light has been shed upon such phenomena as cloud formation, the growth of plants, the opening of blossoms, etc.

\section{AREA AND NUMBER OF SPECIES}

\author{
By DR. C. B. WILLIAMS \\ Rothamsted Experimental Station
}

$F$ R many years there have been discussions of the relation between the size of a sample of an animal or plant community and the number of species contained in it. Until recently, however, most of the approach has been from the botanical side.

So long ago as 1859 Watson $^{1}$ gave a table of the area and number of species of flowering plants in each of the 18 provinces and 38 sub-provinces into which he divided Great Britain, and commented on the fact that one square mile of diversified country in the north of Surrey contained nearly half the species of plants of the whole 760 square miles of the county. Much later, Willis ${ }^{2}$ touched on the same problem from a slightly different angle, as he was more interested in the frequency of species with different distributions than with the actual number of species in one area. The question was then taken up by Arrhenius, Gleason and others by the use of the quadrat system of examining very carefully all the plants on a number of quite small areas as a random sample of the whole.

Arrhenius $^{3}$ considered that the relation between area $(A)$ and number of species of plants $(S)$ on various sizes of plots in the same botanical association was given by the formula $A$ varies as $(S)^{n}$ where $n$ is a constant for the population under consideration. Rich types (that is, associations rich in species) have a low $n$ value and poor types a high value, but the range of $n$ was quite small. This relation should give 